\title{
Evidence for Cognitive Deficits in X-Linked Charcot-Marie-Tooth Disease
}

\author{
Dimitrios Kasselimis $^{1,2, *}$ (D) , Georgia Karadima ${ }^{3}$, Georgia Angelopoulou ${ }^{1}$, Marianthi Breza ${ }^{3}$, Dimitrios Tsolakopoulos ${ }^{1}$, \\ Constantin Potagas ${ }^{1}$, Marios Panas ${ }^{3}$ and Georgios Koutsis ${ }^{3}$ \\ ${ }^{1}$ Neuropsychology and Language Disorders Unit, 1st Department of Neurology, Eginition Hospital, School of Medicine, National and Kapodistrian University \\ of Athens, 11528 Athens Greece \\ ${ }^{2}$ Division of Psychiatry and Behavioral Sciences, School of Medicine, University of Crete, Greece \\ ${ }^{3}$ Neurogenetics Unit, 1st Department of Neurology, Eginition Hospital, School of Medicine, National and Kapodistrian University of Athens, Greece
}

(Received January 28, 2019; Final Revision September 3, 2019; AcCePted September 16, 2019; First Published Online January 17, 2020)

\begin{abstract}
Objective: X-linked Charcot-Marie-Tooth disease (CMTX) is an hereditary neuropathy caused by mutations in GJB1 coding for connexin-32, found in Schwann cells, but also expressed in oligodendrocytes. Reports have identified CNS involvement in CMTX, but no systematic study of cognitive function has been published. Methods: We assessed 24 CMTX patients (13 males; 9 GJB1 mutations) with a comprehensive neuropsychological battery, including tests of memory, language, and executive functions. Results: No differences in cognitive performance were observed between males and females. A case-by-case investigation revealed selective deficits in individual patients. One subgroup (29\%) demonstrated executive abnormalities; and a non-overlapping subgroup (29\%), prominent reading (decoding) abnormalities. Conclusions: The present data provide evidence for cognitive deficits in CMTX. Emerging neuropsychological patterns are also discussed.
\end{abstract}

Keywords: Executive functions, Cognitive flexibility, Reading fluency, Decoding, Connexin-32, GJB1, Cognitive impairment, CNS involvement

\section{INTRODUCTION}

X-linked Charcot-Marie-Tooth disease (CMTX), a hereditary sensorimotor neuropathy, is caused by mutations in GJB1 coding for connexin-32 (Cx-32). Cx-32 is a gap-junction protein expressed in peripheral Schwann cells, but also found in oligodendrocytes within the central nervous system (CNS) (Scherer \& Kleopa, 2012).

CMTX constitutes around $7-15 \%$ of all CMTs, which is one of the commonest inherited neuromuscular diseases, with an overall population prevalence of around 1 in 2500 (Rossor, Polke, Houlden, \& Reilly, 2013; Wang \& Yin, 2016). In the Greek population, CMTX has been estimated at around 5\% of CMT (Karadima, Floroskufi, Koutsis, Vassilopoulos, \& Panas, 2011). The overall prevalence of CMTX in Greece can, therefore, be estimated at around 2 in 100,000.

*Correspondence and reprint requests to: Dimitrios Kasselimis, Neuropsychology and Language Disorders Unit, 1st Department of Neurology, Eginition Hospital, National and Kapodistrian University of Athens, 74 Vas. Sofias Avenue, 11528 Athens, Greece. E-mail: dkasselimis@gmail.com
Several reports have identified CMTX patients with CNS involvement that can range from a transient encephalopathy to asymptomatic white matter abnormalities on brain MRI. Studies presenting MRI findings of individual patients have revealed subcortical white matter involvement, either transient and more severe in concomitance with CNS symptoms, or permanent and milder during asymptomatic periods. White matter involvement has often shown a predilection for posterior subcortical regions, usually involving the splenium of the corpus callosum (Karadima et al., 2014; Panas, Kalfakis, Karadimas, \& Vassilopoulos, 2001; Paulson et al., 2002). A recent case series of CMTX patients that looked at brain MRI findings in a more systematic fashion identified hyperintensity in the splenium of the corpus callosum in $>50 \%$ of cases, significantly higher compared to controls (Koutsis et al., 2019). Additionally, a recent systematic study of diffusion tensor imaging (DTI) in CMT revealed characteristic brain DTI abnormalities in patients with CMTX most prominent posteriorly and in the corpus callosum (Lee et al., 2017). Such anatomical abnormalities are expected to reflect on cognitive functions, given the established role of commissural fibers such as the 
corpus callosum on cognition (Hinkley et al., 2012; Zahr, Rohlfing, Pfefferbaum, \& Sullivan, 2009). Callosal fibers are known to be involved in cognition, in the sense that they allow interhemispheric connections, which support bilateral information processing, especially during highly demanding tasks. A plethora of studies provides evidence in favor of the involvement of the corpus callosum in several cognitive domains, such as visuospatial attention, higher-level visual processing, and language (for a review, see Kasselimis \& Nidos, 2015).

In patients with CMTX, Lee et al. (2017) found additional abnormalities (indicated by decreased fractional anisotropy and/or increased radial diffusivity) in several white matter tracts, including the external capsule, the cingulate, the fornix, and the superior longitudinal fasciculus (SLF I). The external capsule is known to consist of fibers mainly supporting cortico-subcortical connections (Kumral \& Çalli, 2006; Ribas, Yağmurlu, de Oliveira, Ribas, \& Rhoton, 2018). Most importantly, it is often indistinguishable from the extreme capsule in studies utilizing standard neuroradiological evaluation (e.g. Efthymiopoulou et al., 2017) but also advanced neuroimaging methodologies (e.g. Forkel et al., 2014; for a discussion, see Dick \& Tremblay, 2012). The extreme capsule fasciculus has been shown to support cortico-cortical connections between prefrontal and temporal association areas (Petrides, 2014). The same stands for SLF I, which facilitates communication between the superior parietal lobule and the caudal dorsolateral and dorsomedial frontal cortex (Petrides, 2014). Such cortico-cortical connections between association/multisensory cortical regions are thought to support the integration of information during higher-level processing, and, more specifically, cognitive functions, including components of working memory and executive functions in general, are shown to engage a fronto-parietal network (Champod \& Petrides, 2007, 2010; Rottschy et al., 2012; Wager \& Smith, 2003).

The cingulate cortex is suggested to be involved in various cognitive processes (Bush, Luu, \& Posner, 2000). In particular, the anterior cingulate cortex has been associated with executive control (Carter et al., 2000; Markela-Lerenc et al., 2004) and the posterior cingulate cortex has been shown to be involved in memory, internally directed thought, and attentional focus (Leech \& Sharp, 2013; Vogt, Finch, \& Olson, 1992). Finally, the fornix allows communication between the hippocampus and other brain regions (Nieuwenhuys, Voogd, \& van Huijzen, 2008, and therefore may be related to memory consolidation.

In addition to clinical and brain MRI data, older evoked potential studies have also identified CNS involvement in families with CMTX (Nicholson \& Corbett, 1996; Zambelis et al., 2008). In fact, CMTX is often thought of as both a peripheral nervous system and CNS disease (Abrams \& Freidin, 2015; Abrams \& Scherer, 2012). It is, therefore, highly plausible that cognitive function may be affected in these patients. Any potential cognitive deficits would further disable CMTX patients in their everyday life, by adding insult to injury already caused by peripheral motor and sensory impairment, which can affect both upper and lower extremities (Pareyson \& Marchesi, 2009).

Despite the aforementioned evidence, no systematic study of cognitive function in patients with CMTX has been reported to date. In a single family report, episodic memory loss and cognitive impairment, suggestive of probable Alzheimer's disease, was identified in two sisters and their brother respectively (Stancanelli et al., 2012). The only detailed neuropsychological study on CMT patients involved a case series of 30 patients with CMT type 1A (CMT1A) and hereditary neuropathy with liability to pressure palsies (HNPP), different subtypes of CMT than CMTX. CMT1A and HNPP represent the commonest form of CMT, are due to copy number variation in the peripheral myelin protein 22 gene (PMP22), and have a combined population prevalence that can be as high as 10 times that of CMTX (Chanson et al., 2013; van Paassen et al., 2014). It is noteworthy that a priori evidence of CNS involvement for CMT1A and HNPP is very limited compared to CMTX, given that peripheral myelin protein 22 is almost exclusively expressed in the peripheral nervous system (van Paassen et al., 2014).

The above provides indications for CNS involvement in CMTX and further raise the possibility of cognitive impairment in such patients. Presently, we attempted to systematically assess cognitive function in patients with CMTX, using a comprehensive neuropsychological battery. Since relevant evidence is limited in the literature, we decided to include a wide variety of psychometric measures corresponding to several cognitive domains. In particular, verbal memory and learning refer to encoding, short-term retention, and consolidation of verbal information. Verbal and visuospatial working memory refer to the ability to temporarily retain and manipulate verbal and visuospatial stimuli, respectively. Investigation of verbal and language skills included confrontation naming and receptive vocabulary, both involving access and retrieval of lexical-semantic representations. We also examined auditory comprehension, through a complex command task. Assessment of executive functions included tests evaluating processing speed, that is, the ability to process information to complete a complex task within a particular timeframe, and cognitive flexibility, a psychological construct which reflects the ability to switch between strategies, generally described as shifting. Moreover, we assessed inhibition, which is described as the ability to suppress a spontaneous, overlearned ability and replace it with another one based on task requirements. Finally, visual attention was assessed within the framework of executive functions as the ability to scan and focus on specific visual stimuli (for a detailed description of cognitive functions, see Mesulam, 2000; for a comprehensive presentation of various neuropsychological measures, see Lezak, Howieson, Loring, \& Fischer, 2012).

\section{METHODS}

The Neurogenetics Unit at the 1st Department of Neurology, Eginition Hospital, Athens, Greece, has provided molecular 


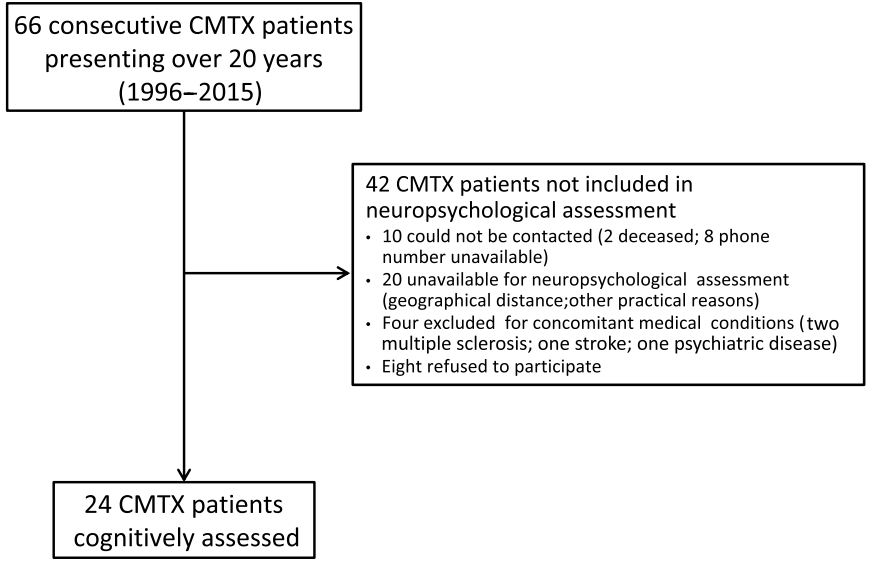

Fig. 1. Flow diagram illustrating recruitment of patients for neuropsychological assessment from a total cohort of CMTX patients presenting over 20 years.

diagnostic testing for CMTX to patients from all regions of the country, acting effectively as a national reference center. In total, 66 patients with mutations in $G J B 1$ have been identified over two decades. Of these, 56 patients could be contacted by phone to request participation in the present investigation. After excluding patients with concomitant medical conditions, such as alcohol or drug abuse; epilepsy; severe cranial trauma; autoimmune, vascular or other systemic disease; as well as patients with clinical evidence of visual or hearing impairment that could affect cognitive function, 24 agreed to participate (Chanson et al., 2012). Patient recruitment is summarized in Figure 1. The study was approved by the hospital ethics committee, and informed consent was obtained from all patients.

In total, 24 patients with molecularly confirmed CMTX were included $(13$ males; mean age $=43.4 \pm 10.3$ years, range 19-61 years; mean education $=14.0 \pm 3.3$ years, range 6-18; nine different GJB1 mutations). All patients were examined neurologically by the neurologists of the Neurogenetics Unit. Clinical and electrophysiological findings were used to calculate the CMT neuropathy score (Shy et al., 2005). Following exclusion of the PMP22 duplication, all cases were Sanger-sequenced for mutations in $G J B 1$, as previously described (Karadima et al., 2014).

All patients were assessed by an experienced neuropsychologist according to standard administration procedures described in the corresponding normative studies (see below). Neuropsychological testing included the Greek version of the Auditory Verbal Learning Test (AVLT) (Constantinidou, Christodoulou, \& Prokopiou, 2014), the Boston Naming Test (BNT; Kaplan, Goodglass, \& Weintraub, 1983), and the Peabody Picture Vocabulary Test-Revised (PPVT-R; Dunn, 1981), both standardized in Greek (Simos, Kasselimis, \& Mouzaki, 2011a, 2011b), for assessing access to mental lexicon, the Comprehension of Instructions in Greek (CIG; Simos, Kasselimis, Potagas, \& Evdokimidis, 2014), the Controlled Oral Word Fluency (COWF; Kosmidis, Vlahou, Panagiotaki, \&
Kiosseoglou, 2004), and two standardized word and pseudoword reading fluency measures. The reading fluency task consists of a list of 112 high-frequency words in order of increasing length. The pseudoword fluency task consists of a list of 70 pseudowords in order of increasing length. For both tasks, the patient is asked to read items aloud with a time limit of $45 \mathrm{~s}$, as fast as possible. Total number of correctly read items within the given time limit is the final score of the patient in each task (for a detailed description of the psychometric tool, see Simos, Sideridis, Kasselimis, \& Mouzaki, 2013). A Digit Span task (DS; Simos, Papastefanakis, Panou, \& Kasselimis, 2011) and the Corsi Block-Tapping task (Corsi, 1972; Kessels, Van Den Berg, Ruis, \& Brands, 2008) were used for verbal and visual working memory, respectively. For frontal and executive functions, Trail Making Test (TMT; Zalonis et al., 2007), Symbol Digit Modality Test (SDMT; Constantinidou et al., 2012), and Stroop Test (Zalonis et al., 2009) were used. It should be noted that in order to prevent the confounding ation of movement deficits, additional scores (TMT B-A and TMT B/A) were calculated for each patient (Christidi, Kararizou, Triantafyllou, Anagnostouli, $\&$ Zalonis, 2015). Impaired performance was defined on the basis of either the fifth percentile or -1.5 standard deviation criteria, depending on the available normative data. Statistical analysis was performed on SPSS, version 20.

\section{RESULTS}

Genetic, demographic, clinical, and neuropsychological data for all 24 individual patients are presented in Table 1. Individual data on performance ( $\mathrm{Z}$ scores and percentiles) in the neuropsychological tests are shown in Table 2. No significant difference in CMT neuropathy score was observed between male and female patients (12.4 vs. 9.3, $p=.14$; Mann-Whitney U test). We specifically display neuropsychological tests with abnormal results for individual patients. Initially, we performed group comparisons using KruskalWallis, with gender, mutation location, and specific mutations (analysis limited to mutations carried by at least two patients) as independent variables. Results indicated that there were no significant differences between groups with regard to scores on neuropsychological tests (first analysis: males vs. females; second analysis: extracellular vs. intracellular vs. intramembrane location; third analysis: R164Q vs. V120E vs. V37L vs. P70F vs. T55I).

We then transformed raw scores to $\mathrm{Z}$ scores/percentiles based on the corresponding norms for each test. A case-bycase investigation revealed that there were several patients displaying specific cognitive deficits. Three non-overlapping groups emerged based on patterns of performance on the neuropsychological battery. Group 1 (seven patients, 29\%) demonstrated prominent executive deficits (as indicated by the TMT B/A index; at least $1.5 \mathrm{SD}$ below mean healthy performance); Group 2 (seven patients, 29\%) demonstrated prominent deficits in decoding written stimuli with intact 
Table 1. Genetic, demographic, clinical and neuropsychological data of individual patients with CMTX studied with a comprehensive neuropsychological battery

\begin{tabular}{|c|c|c|c|c|c|c|c|c|c|c|}
\hline No & $\begin{array}{l}\text { Mutation in } \\
\text { Cx-32 }\end{array}$ & $\begin{array}{l}\text { Mutation } \\
\text { location }\end{array}$ & Family & Age & Gender & Edu & $\begin{array}{l}\text { CMT } \\
\text { score }\end{array}$ & CNS signs & $\begin{array}{l}\text { Specific cognitive } \\
\text { deficits }\end{array}$ & $\begin{array}{l}\text { Cognitive } \\
\text { group }\end{array}$ \\
\hline 2 & V120E & IC & 8 & 59 & $\mathrm{~F}$ & 12 & 9 & No & $\mathrm{EX}-\mathrm{CF}$ & 1 \\
\hline 4 & V120E & IC & 8 & 39 & M & 15 & 15 & $\begin{array}{l}\text { Extensor } \\
\text { plantars }\end{array}$ & EX-CF & 1 \\
\hline 7 & T55I & $\mathrm{EC}$ & 3 & 59 & M & 16 & 24 & No & EX-CF, WM-V, EM-DR & 1 \\
\hline 9 & H100Q & IC & 7 & 19 & M & 13 & 4 & Brisk reflexes & EX-CF, EX-I, L-N, S-F & 1 \\
\hline 10 & R164Q & $\mathrm{EC}$ & 11 & 44 & M & 18 & 12 & No & EX-CF & 1 \\
\hline 12 & R164Q & $\mathrm{EC}$ & 12 & 39 & M & 16 & 10 & No & $\mathrm{EX}-\mathrm{CF}$ & 1 \\
\hline 24 & $\mathrm{R} 22 \mathrm{X}$ & IM & 1 & 39 & M & 17 & 9 & No & $\mathrm{EX}-\mathrm{CF}$ & 1 \\
\hline 1 & V120E & IC & 8 & 52 & $\mathrm{~F}$ & 8 & 14 & No & $\begin{array}{l}\text { L-D, EX-PS, WM-VS, } \\
\text { EM-E }\end{array}$ & 2 \\
\hline 5 & P70F & $\mathrm{EC}$ & 6 & 40 & $\mathrm{~F}$ & 13 & 25 & No & L-D, EM-LC & 2 \\
\hline 8 & P70F & $\mathrm{EC}$ & 6 & 42 & $\mathrm{~F}$ & 16 & 14 & No & L-D, EX-I, L-RV, S-F & 2 \\
\hline 17 & V120E & IC & 8 & 52 & $\mathrm{~F}$ & 7 & 11 & No & L-D; EM-E & 2 \\
\hline 18 & R164Q & $\mathrm{EC}$ & 13 & 42 & M & 12 & 14 & No & L-D & 2 \\
\hline 20 & R164Q & $\mathrm{EC}$ & 12 & 36 & M & 15 & 14 & No & L-D & 2 \\
\hline 22 & Y154X & $\mathrm{EC}$ & 9 & 48 & M & 15 & 12 & No & $\begin{array}{l}\text { L-D, L-C, L-RV, L-N, } \\
\text { S-F }\end{array}$ & 2 \\
\hline 3 & R164Q & $\mathrm{EC}$ & 10 & 38 & M & 18 & 9 & No & None & 3 \\
\hline 6 & V120E & IC & 8 & 34 & $\mathrm{~F}$ & 12 & 9 & $\begin{array}{l}\text { Extensor } \\
\text { plantars }\end{array}$ & WM-VS & 3 \\
\hline 11 & R164Q & $\mathrm{EC}$ & 12 & 57 & $\mathrm{~F}$ & 6 & 3 & No & None & 3 \\
\hline 13 & R164Q & $\mathrm{EC}$ & 11 & 61 & $\mathrm{~F}$ & 16 & 1 & No & None & 3 \\
\hline 14 & V120E & IC & 8 & 34 & $\mathrm{~F}$ & 16 & 4 & No & None & 3 \\
\hline 15 & T55I & $\mathrm{EC}$ & 4 & 31 & M & 17 & 15 & No & None & 3 \\
\hline 16 & V37L & IM & 2 & 47 & $\mathrm{~F}$ & 14 & 2 & No & None & 3 \\
\hline 19 & R164Q & $\mathrm{EC}$ & 14 & 34 & M & 16 & 7 & $\begin{array}{l}\text { Extensor } \\
\text { plantars }\end{array}$ & L-C & 3 \\
\hline 21 & V37L & $\mathrm{IM}$ & 2 & 42 & $\mathrm{~F}$ & 17 & 10 & No & None & 3 \\
\hline 23 & C64Y & $\mathrm{EC}$ & 5 & 53 & M & 12 & 16 & No & S-F & 3 \\
\hline
\end{tabular}

cx-32, connexin-32; Edu, education; CMT score, CMT neuropathy score; IM, intramembrane; EC, extracellular; IC, intracellular; EM-DR, Episodic MemoryDelayed Recall, corresponding to AVLT delayed recall subscale; EM-E, Episodic Memory Encoding, corresponding to AVLT immediate recall subscale; EM-LC, Episodic Memory-Learning Curve, corresponding to AVLT learning index; EX-CF, Executive Functions-Cognitive Flexibility, corresponding to TMT B/A index; EX-I, Executive Functions-Inhibition, corresponding to Stroop-CW subscale; EX-PS, Executive Functions-Processing Speed, corresponding to SDMT index; L-C, Language-Comprehension of complex commands, corresponding to CIG index; L-D, Language-Decoding, corresponding to pseudoword reading fluency index; L-N, Language-Naming, corresponding to BNT index; L-RV, Language-Receptive Vocabulary, corresponding to PPVT index; S-F, Semantic Fluency; WM-V, Working Memory-Verbal, corresponding to Digit Span index; WM-VS, Working Memory-Visuospatial, corresponding to Corsi Block-Tapping task.

word recognition ability (as indicated by performance on the two reading fluency tasks; at least $1.5 \mathrm{SD}$ below mean healthy performance on the pseudoword fluency task and over -1.5 SD for the word fluency task); and Group 3 (10 patients, $42 \%$ ) demonstrated either non-specific, sparse deficits, or intact cognitive functions. More specifically, one patient demonstrated lower-than-expected performance on the forward condition of the Corsi Block-Tapping task; one patient exhibited lower-than-expected performance on CIG; and one patient scored lower than expected on the semantic fluency subscale of COWF. The rest of the patients included in Group 3 did not demonstrate any cognitive deficit (see Table 1). Inclusion criteria for the third group were scores above $-1.5 \mathrm{SD}$ for the TMT B/A index and the pseudoword fluency task. The three groups were matched for age, years of formal schooling (as indicated by non-significant
Kruskal-Wallis results), and gender (as indicated by non-significant chi-square results). No differences were observed between groups with regard to mutation location, as indicated by chi-square analysis. In order to statistically assess whether the percentage of patients demonstrating impaired performance could be attributed to chance, we conducted statistical analyses with the binomial test, which is actually a chi-square test for goodness of fit. The question was whether the percentage of patients found impaired in a single neuropsychological measure (i.e. $29 \%$ corresponding to each of the aforementioned subgroups) is different from the expected percentage of healthy participants scoring below 1.5 SD in a hypothesized normal distribution of normative data, which is equal to $7 \%$. Results showed that the percentages of patients demonstrating impaired performance in decoding 
Table 2. Individual data for the neuropsychological battery: $\mathrm{Z}$ scores and percentiles

\begin{tabular}{|c|c|c|c|c|c|c|c|c|c|c|c|c|c|c|c|c|c|c|c|}
\hline No & AVLT-1-5 & AVLT-LC & AVLT-7 & FL-PH & FL-SEM & TMT-A & TMT-B & TMT B/A & SDMT & PPVT-R & BNT & CIG & RW & RpsW & DS-F & DS-B & C-F & C-B & SNST \\
\hline 2 & $>25$ th & 50th & 50th & -.3 & -.3 & 1.9 & -4.0 & -4.0 & -1.0 & $>25$ th & 50 th & .3 & .6 & .6 & 50 th & 50 th & -.1 & -.3 & 1.8 \\
\hline 4 & 50th & 25 th & 25 th & -.4 & 1.5 & 1.0 & .2 & -1.6 & -.3 & 15 th & $>25$ th & .7 & 1.3 & -.2 & $>50$ th & $>25$ th & 2.5 & -.3 & - \\
\hline 7 & $>15$ th & 50 th & 5 th & -.6 & -.8 & -.7 & -4.0 & -3.1 & -1.0 & 15 th & $>25$ th & -.8 & -.8 & -.8 & 25 th & 5 th & 1.1 & -.3 & .1 \\
\hline 9 & $>25$ th & $>50$ th & 50th & .5 & -2.7 & .3 & -1.6 & -2.3 & -.3 & - & $<5 \%$ & -.4 & .5 & -.1 & $>5$ th & 50 th & 3.1 & -.3 & -5.2 \\
\hline 10 & $>25$ th & $>50$ th & 25 th & .0 & -.6 & -.6 & -3.2 & -1.7 & -.5 & 10th & $>25$ th & .7 & .4 & .5 & $>50$ th & $>25$ th & 1.7 & 1.0 & -.7 \\
\hline 12 & 50th & $>25$ th & 50 th & 1.2 & .0 & .2 & -1.5 & -2.0 & .0 & $>5$ th & $>25$ th & 1.2 & 1.0 & .5 & $>5$ th & 50th & -.9 & -.3 & -.7 \\
\hline 24 & $>50$ th & $>50$ th & $>50$ th & -.4 & .9 & 1.0 & -.3 & -2.4 & -.3 & $>25$ th & $>75$ th & .7 & -.8 & -.4 & $>50$ th & $>50$ th & 2.5 & .6 & .4 \\
\hline 1 & $<5$ th & $>50$ th & 50th & - & .0 & -1.6 & -2.9 & -.5 & -1.5 & $>50$ th & 75 th & -.2 & -1.1 & -2.1 & $>25$ th & $>5$ th & 2.5 & -1.5 & 1.6 \\
\hline 5 & $>25$ th & $<5 \%$ & $>25$ th & -1.4 & 1.2 & .4 & 1.1 & .7 & -.8 & 50 th & $>15$ th & .7 & -1.2 & -1.6 & 25 th & $>50$ th & 1.1 & .6 & - \\
\hline 8 & $>25$ th & $>50 \%$ & 25 th & 1.4 & -4.0 & .3 & -.3 & -.8 & -1.0 & $<5$ th & $>15$ th & .7 & -1.3 & -4.1 & 25 th & $>25$ th & 3.1 & .6 & -3.6 \\
\hline 17 & $<5$ th & 50 th & $>15$ th & -.9 & .9 & -4.0 & -3.8 & 1.4 & -1.0 & $>25$ th & 75 th & -.2 & -.9 & -1.5 & $>25$ th & $>25$ th & -.5 & -.6 & .8 \\
\hline 18 & 50th & $>50 \%$ & 25 th & -.8 & .6 & -.4 & -.3 & .5 & 1.5 & $50 \%$ & $>75$ th & -.7 & -.4 & -1.5 & $>50$ th & $>50$ th & 1.7 & 1.0 & .6 \\
\hline 20 & 50 th & $>50 \%$ & 50th & 1.2 & .1 & .5 & .0 & -.8 & 1.0 & $>25$ th & $>75$ th & 1.2 & .8 & -1.7 & $>25$ th & 50 th & 3.4 & .6 & .4 \\
\hline 22 & $>25$ th & 50th & $>50$ th & -1.0 & -2.1 & -2.6 & -7.0 & -1.2 & - & $<5$ th & $<5$ th & -2.0 & -1.2 & -2.3 & $>5$ th & 25 th & 1.1 & 2.7 & - \\
\hline 3 & 50 th & $>50$ th & 50 th & -1.1 & .4 & -.7 & -.9 & -.1 & .3 & 10th & $>20$ th & -.9 & .7 & -.4 & $>50$ th & $>50$ th & 1.7 & -.2 & .6 \\
\hline 6 & $>50$ th & $>50$ th & $>50$ th & -.7 & .7 & -1.0 & -1.7 & .1 & -1.0 & 20th & 50th & -.2 & 1.4 & 1.5 & $>50$ th & 50th & -1.5 & .2 & 1.1 \\
\hline 11 & 50th & $>50$ th & $>50$ th & -.3 & -.6 & -1.2 & -4.2 & -1.0 & -.5 & 80th & 80th & .9 & .3 & 1.0 & 50 th & $>50$ th & 1.7 & 1.0 & 1.3 \\
\hline 13 & $>25$ th & $>50$ th & $>25$ th & .0 & -1.2 & -.4 & -2.3 & -1.4 & -1.0 & $>25$ th & $>75$ th & .7 & .7 & .1 & 25 th & $>5$ th & .9 & 1.0 & .2 \\
\hline 14 & $>50$ th & 50th & $>50$ th & -1.0 & .2 & .6 & 1.5 & .6 & .4 & 25 th & $>25$ th & .1 & 1.7 & 3.1 & 50 th & $>50$ th & 3.1 & 3.3 & .6 \\
\hline 15 & $>50$ th & $>50$ th & $>50 \%$ & 1.4 & 1.2 & -2.5 & -2.5 & .4 & .3 & $>20$ th & $>25$ th & 1.2 & -.3 & .7 & $>50$ th & $>50$ th & 3.7 & 3.3 & .6 \\
\hline 16 & $>50 \%$ & $>25$ th & 50th & .2 & 1.6 & -1.9 & -1.3 & .7 & -.5 & 50 th & $>20$ th & -.4 & -.1 & 1.0 & $>5$ th & 25 th & 2.5 & .6 & -.7 \\
\hline 19 & $>25$ th & 50th & 15 th & -.6 & -.8 & -.9 & -1.1 & .0 & -.7 & 50th & $>75$ th & -2.5 & 1.6 & 2.2 & $>50$ th & $>50$ th & 4.8 & 1.6 & .6 \\
\hline 21 & $>50$ th & 50 th & $>25$ th & -.3 & 2.0 & .0 & .8 & .7 & .1 & 50 th & $>80$ th & 1.2 & .2 & .8 & 50th & $>50$ th & 4.8 & 2.7 & .9 \\
\hline 23 & $>25$ th & $>50$ th & 25 th & .7 & -1.5 & -.3 & -3.0 & -.7 & -.5 & $>75$ th & $>20$ th & .3 & 1.4 & 2.6 & $>50$ th & $>50$ th & 1.1 & .6 & 1.8 \\
\hline
\end{tabular}

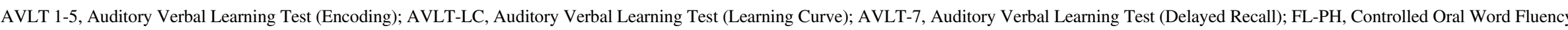

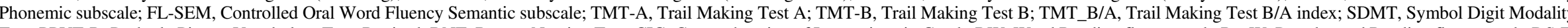

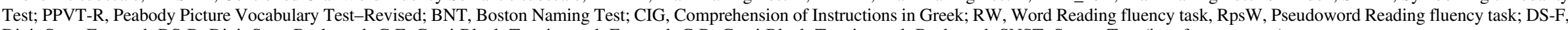
Digit Span Forward; DS-B, Digit Span Backward; C-F, Corsi Block-Tapping task Forward; C-B, Corsi Block-Tapping task Backward; SNST, Stroop Test (interference score). 
and cognitive flexibility are significantly $>7 \%(p=.001)$, and therefore cannot be attributed to chance. Binomial tests for the rest of the neuropsychological measures did not yield any significant results.

In order to investigate the possibility that the differences observed in cognitive measures were due to the severity of neuropathy, we then decided to assess the effect of the degree of neuropathy on neuropsychological measures. First, we conducted correlation analyses between the CMTX neuropathy score and performance on the pseudoword reading fluency task, as well as the TMT B/A index. Results were negative, since Spearman rho analyses failed to yield significant results. In particular, the correlation coefficient between TMT B/A index and neuropathy score was $-.179(p=.402)$ and the correlation coefficient between pseudoword reading fluency score and neuropathy score was $-.343(p=.101)$. Second, we compared the three groups with regard to CMTX neuropathy score, in the sense that a difference in favor of the non-impaired group could raise the possibility of the cognitive deficits being due to the severity of neuropathy. A Kruskal-Wallis test yielded a marginally significant result; $\chi^{2}(2)=6.215, p=.045$. However, follow-up pairwise comparisons, implementing a Bonferroni-adjusted significance level at $\alpha=.017$, failed to show significant differences between any pair of groups: $U=13.5, p=.154$ for the comparison between Group 1 and Group 2; $U=20.5, p=.154$ for the comparison between Group 1 and Group 3; $U=12$, $p=.024$ for the comparison between Group 2 and Group 3 .

\section{DISCUSSION}

We presently aimed to investigate the cognitive function of patients with CMTX. Despite the well-described CNS involvement that can be observed in these patients, cognition has not been previously investigated, and the available literature is limited to a single family report. In that report, an unspecified neuropsychological battery revealed episodic memory loss in two sisters, and episodic and semantic memory loss, constructional apraxia, alexia, agraphia, and acalculia in their brother (siblings aged 53-62 years) (Stancanelli et al., 2012). To our knowledge, this is the first study investigating multiple cognitive domains in patients with CMTX by implementing a comprehensive neuropsychological battery.

The most interesting finding to emerge was the delineation of three non-overlapping groups, one with prominent executive deficits (Group 1), one with prominent pseudoword reading deficits (Group 2), and one with non-specific or no cognitive deficits (Group 3). Regarding Group 1, the TMT $\mathrm{B} / \mathrm{A}$ index is thought to be a reliable measure of cognitive flexibility (Christidi et al., 2015). The low TMT B/A score in some CMTX patients may thus reflect underlying frontal dysfunction. Although the general consensus is that the score relies heavily on prefrontal activation, recent findings indicate that TMT performance is related to posterior and anterior cortical regions and may, in fact, reflect more general cognitive ability (Chan et al., 2015; Zakzanis et al., 2005). The reading deficits observed in Group 2 are particularly interesting in light of the predominance of posterior DTI abnormalities in some CMTX patients and the known involvement of posterior brain systems in reading (Lee et al., 2017; Shaywitz et al., 2002). According to the widely accepted Dual-Route Model (Coltheart, Curtis, Atkins, \& Haller, 1993), there are two independent mechanisms involved in reading. The first one, corresponding to the lexical route, is activated in expert readers, during recognition and production of real words, via access to input and output lexical/semantic representations (where the visual form and the right phonological/ spoken form of words already familiar to the individual speaker are stored). The second one, corresponding to the sublexical route, is engaged in phonological decoding of novel printed verbal stimuli via grapheme-to-phoneme conversion. The latter route is thought to be activated during pseudoword reading, by mapping individual orthographic aspects to their phonological correlates, thus integrating visual information into a phonological representation of any given meaningless string of graphemes. Based on the above, Group 2 demonstrates an extremely specific cognitive impairment, revealing a distinct pattern with prominent difficulty in decoding written stimuli, with intact word recognition capacity. Brain imaging findings suggest that the left temporoparietal region is the neural substrate of phonological decoding (Simos et al., 2002). It is also noteworthy that, in this context, the lexical route takes the ventral pathway as opposed to the dorsal pathway. The latter seems to be affected by the disease in Group 2.

We failed to find any association between gender and impairment in any of the cognitive functions examined. This finding may seem prima facie strange, given that males with CMTX have more severe neuropathy than females, CNS involvement has been reported overwhelmingly in male patients, and abnormal DTI findings have been observed exclusively in male patients (Karadima et al., 2014; Lee et al., 2017; Panas et al., 2001; Paulson et al., 2002). However, it is noteworthy that in our cohort the severity of neuropathy did not differ significantly between males and females, indicating that relatively severely affected females had been included. Furthermore, it is possible that different pathogenic mechanisms may underlie peripheral vs. central manifestations of CMTX, the second putatively involving trans-dominant-negative effects on other CNS connexins, which may be differentially influenced by gender (Scherer \& Kleopa, 2012). A whole-sample correlation analysis (including both males and females) showed a rather weak association of decoding deficits with higher CMT neuropathy scores. Although the correlation proved to be non-significant, one could argue that there is a kind of a trend for an inverse association between the decoding skills and severity of neuropathy. However, in addition to lack of statistical significance, the value of coefficient per se does not indicate a strong or even moderate association. It should be also noted that the Kruskal-Wallis analysis indicated a marginally significant difference with regard to CMTX neuropathy score between the three cognitive groups (with Group 2 being inferior 
compared to the other two), but follow-up pairwise comparisons did not survive a Bonferroni correction. In any case, our data imply the existence of an inverse association trend between the degree of neuropathy and the ability to decode non-word printed stimuli. This should be further tested and possibly confirmed by studies with larger patient cohorts, something that does not seem to be possible for the Greek CMTX population, due to its small number.

No effect of mutation location within $\mathrm{Cx}-32$ on cognitive performance was observed. Furthermore, no individual mutation was specifically associated with cognitive impairment, although the relatively small case number per mutation largely precluded such analysis. Evidence for CNS involvement can be found in the literature for at least four mutations included (T55I, H100Q, Y154X, R164Q), and some, though not all, patients carrying these mutations presently showed evidence of cognitive deficits (Fusco et al., 2010; Karadima et al., 2014; Panas et al., 2001; Zambelis et al., 2008).

A detailed neuropsychological study has been recently published in patients with CMT1A and HNPP, both hereditary neuropathies sharing several common features with CMTX with respect to peripheral nerve involvement, with sample sizes comparable to this report and neuropsychological tests that only partially overlapped with the present battery (Chanson et al., 2012). The authors observed cognitive impairment in $70 \%$ of patients with PMP22 mutations, affecting primarily executive functions, working memory, and verbal episodic memory. This observation was accompanied by abnormal imaging findings regarding white matter volume, DTI, and magnetic spectroscopy. DTI abnormalities, however, were at odds with the completely negative findings on DTI recently reported in CMT1A patients (Lee et al., 2017). Cognitive deficits, therefore, seem to be a feature of CMT caused not only by mutations in $\mathrm{Cx}-32$, as presently observed, but also by the more common mutations in $P M P 22$, with some controversy surrounding their relationship with imaging abnormalities. In future studies, it would be important to combine cognitive assessment with imaging parameters in the same subjects with CMTX.

Several limitations to the present study should be specifically stated. The most important is the absence of a control group with a comparable neuropathy. We gave substantial attention to this point during study design. However, there were difficulties that could not be overcome for several clinical reasons. Common metabolic and toxic neuropathies (e.g. alcoholic, diabetic, uremic, due to B1 or B12 deficiency) are caused by agents that commonly result in CNS abnormalities. Additionally, the much rarer autoimmune neuropathies have also been often linked to CNS involvement. These restrictions have also been noted in the paper by Chanson et al. (2012). The obvious choice would have been to have a control group of patients with a more common hereditary neuropathy such as CMT1A. However, the findings by Chanson et al. (2012), suggesting the presence of cognitive involvement in $70 \%$ of these patients, also ruled them out as a satisfactory control group.
A further limitation of this study is the fact that any visual or hearing impairment was only assessed clinically and not neurophysiologically with evoked potentials, during subject screening for exclusion. It is therefore possible, though unlikely, that subjects with minor subclinical involvement of the auditory or visual systems were included in the cohort.

Finally, two further limitations pertaining to this study are the absence of concomitant neuroradiological data that could be correlated to the cognitive abnormalities detected and the absence of a replication of the neuropsychological examination over time with a view to assessing the reversibility, stability, or worsening of any pathological findings. Both these limitations should be addressed in future extensions of the present report.

\section{ACKNOWLEDGEMENTS}

This work has been supported by a grant by Teva Pharmaceuticals (grant no. 12846, special account for research grants, National and Kapodistrian University of Athens). D.K. is supported by IKY Scholarships Programme co-financed by the European Union (European Social Fund) and Greek national funds through the action entitled "Reinforcement of Postdoctoral Researchers," in the framework of the Operational Programme "Human Resources Development Program, Education and Lifelong Learning" of the National Strategic Reference Framework 2014-2020. D.T. is supported by Greece and the European Union (European Social Fund- ESF) through the Operational Programme "Human Resources Development, Education and Lifelong Learning" in the context of the project "Strengthening Human Resources Research Potential via Doctorate Research" (MIS-5000432), implemented by the State Scholarships Foundation (IKY).

\section{CONFLICTS OF INTEREST}

The authors have nothing to disclose.

\section{REFERENCES}

Abrams, C.K. \& Freidin, M. (2015). GJB1-associated X-linked Charcot-Marie-Tooth disease, a disorder affecting the central and peripheral nervous systems. Cell Tissue Research, 360(3), 659-673.

Abrams, C.K. \& Scherer, S.S. (2012). Gap junctions in inherited human disorders of the central nervous system. Biochimica et Biophysica Acta (BBA)-Biomembranes, 1818(8), 2030-2047.

Bush, G., Luu, P., \& Posner, M. (2000). Cognitive and emotional influences in anterior cingulate cortex. Trends in Cognitive Sciences, 4(6), 215-222.

Carter, C., Macdonald, A., Botvinick, M., Ross, L., Stenger, V., Noll, D., \& Cohen, J. (2000). Parsing executive processes: strategic vs. evaluative functions of the anterior cingulate cortex. Proceedings of the National Academy of Sciences, 97(4), 1944-1948.

Champod, A.S., \& Petrides, M. (2007). Dissociable roles of the posterior parietal and the prefrontal cortex in manipulation and 
monitoring processes. Proceedings of the National Academy of Sciences, 104(37), 14837-14842.

Champod, A.S., \& Petrides, M. (2010). Dissociation within the frontoparietal network in verbal working memory: a parametric functional magnetic resonance imaging study. Journal of Neuroscience, 30(10), 3849-3856.

Chan, E., MacPherson, S.E., Robinson, G., Turner, M., Lecce, F., Shallice, T., \& Cipolotti, L. (2015). Limitations of the trail making test part-B in assessing frontal executive dysfunction. Journal of the International Neuropsychological Society, 21(2), 169-174.

Chanson, J., Echaniz-Laguna, A., Blanc, F., Lacour, A., Ballonzoli, L., Kremer, S., Namer, I.J., Lannes, B., Tranchant, C., Vermersch, P., \& de Seze, J. (2013). Central nervous system abnormalities in patients with PMP22 gene mutations: a prospective study. Journal of Neurology, Neurosurgery \& Psychiatry, 84(4), 392-397.

Christidi, F., Kararizou, E., Triantafyllou, N., Anagnostouli, M., \& Zalonis, I. (2015). Derived Trail Making Test indices: demographics and cognitive background variables across the adult life span. Aging, Neuropsychology, and Cognition, 22(6), 667-678.

Coltheart, M., Curtis, B., Atkins, P., \& Haller, M. (1993). Models of reading aloud: dual-route and parallel-distributed-processing approaches. Psychological Review, 100(4), 589-608.

Constantinidou, F., Christodoulou, M., \& Prokopiou, J. (2012). The effects of age and education on executive functioning and oral naming performance in Greek Cypriot adults: the neurocognitive study for the aging. Folia Phoniatrica et Logopaedica, 64(4), 187-198.

Constantinidou, F., Zaganas, I., Papastefanakis, E., Kasselimis, D., Nidos, A., \& Simos, P.G. (2014). Age-related decline in verbal learning is moderated by demographic factors, working memory capacity, and presence of amnestic mild cognitive impairment. Journal of the International Neuropsychological Society, 20(8), 822-835.

Corsi, P.M. (1972). Human memory and the medial temporal region of the brain. Unpublished doctoral dissertation. McGill University, Montreal, Canada.

Dick, A.S. \& Tremblay, P. (2012). Beyond the arcuate fasciculus: Consensus and controversy in the connectional anatomy of language. Brain, 135(12), 3529-3550.

Dunn, L.M. (1981). Manual for the Peabody picture vocabulary test-revised. Circle Pines: American Guidance Service.

Efthymiopoulou, E., Kasselimis, D.S., Ghika, A., Kyrozis, A., Peppas, C., Evdokimidis, I., Petrides, M., \& Potagas, C. (2017). The effect of cortical and subcortical lesions on spontaneous expression of memory-encoded and emotionally infused information: Evidence for a role of the ventral stream. Neuropsychologia, 101, 115-120.

Forkel, S.J., de Schotten, M.T., Kawadler, J.M., Dell'Acqua, F., Danek, A., \& Catani, M. (2014). The anatomy of fronto-occipital connections from early blunt dissections to contemporary tractography. Cortex, 56, 73-84.

Fusco, C., Frattini, D., Pisani, F., Spaggiari, F., Ferlini, A., \& Della Giustina, E. (2010). Coexistent central and peripheral nervous system involvement in a Charcot-Marie-Tooth syndrome X-linked patient. Journal of Child Neurology, 25(6), 759-763.

Hinkley, L.B., Marco, E.J., Findlay, A.M., Honma, S., Jeremy, R.J., Strominger, Z., Bukshpun, P., Wakahiro, M., Brown, W.S., Paul, L.K., Barkovich, A.J., Mukherjee, P., Nagarajan, S.S., \& Sherr, E.H. (2012). The role of corpus callosum development in functional connectivity and cognitive processing. PLoS One, 7(8), e39804. https://doi.org/10.1371/journal.pone.0039804.

Kaplan, E.F., Goodglass, H., \& Weintraub, S. (1983). The Boston naming test, 2nd ed. Philadelphia: Lea \& Febiger.

Karadima, G., Floroskufi, P., Koutsis, G., Vassilopoulos, D., \& Panas, M. (2011). Mutational analysis of PMP22, GJB1 and MPZ in Greek Charcot-Marie-Tooth type 1 neuropathy patients. Clinical Genetics, 80(5), 497-499.

Karadima, G., Koutsis, G., Raftopoulou, M., Floroskufi, P., Karletidi, K.M., \& Panas, M. (2014). Four novel connexin 32 mutations in X-linked Charcot-Marie-Tooth disease. Phenotypic variability and central nervous system involvement. Journal of the Neurological Sciences, 341(1), 158-161.

Kasselimis, D.S. \& Nidos, A. In J.D. Wright (2015). Interhemispheric Interaction in language and cognitive processes, In (editor-in-chief), International encyclopedia of the social \& behavioral sciences, 2nd ed., Vol. 12 (pp. 416-424). Oxford: Elsevier.

Kessels, R.P.C., Van Den Berg, E., Ruis, C., \& Brands, A.M.A. (2008). The backward span of the corsi block-tapping task and its association with the WAIS-III digit span. Assessment, 15(4), 426-434.

Kosmidis, M.H., Vlahou, C.H., Panagiotaki, P., \& Kiosseoglou, G. (2004). The verbal fluency task in the Greek population: normative data, and clustering and switching strategies. Journal of the International Neuropsychological Society, 10(2), 164-172.

Koutsis, G., Breza, M., Velonakis, G., Tzartos, J., Kasselimis, D., Kartanou, C., Karavasilis, E., Tzanetakos, D., Anagnostouli, M., Andreadou, E., Evangelopoulos, M.E., Kilidireas, C., Potagas, C., Panas, M., \& Karadima, G. (2019). X linked Charcot-Marie-Tooth disease and multiple sclerosis: emerging evidence for an association. Journal of Neurology Neurosurgery and Psychiatry, 90(2), 187-194.

Kumral, E. \& Çalli, C. (2006). External and extreme capsular stroke: clinical, topographical and etiological patterns. Cerebrovascular Diseases, 21(4), 217-222.

Lee, M., Park, C.H., Chung, H.K., Kim, H.J., Choi, Y., Yoo, J.H., Yoon, Y.C., Hong, Y.B., Chung, K.W., Choi, B.O., \& Lee, H.W. (2017). Cerebral white matter abnormalities in patients with charcot-marie-tooth disease. Annals of Neurology, 81(1), 147-151.

Leech, R. \& Sharp, D. (2013). The role of the posterior cingulate cortex in cognition and disease. Brain, 137(1), 12-32.

Lezak, M.D., Howieson, D.B., Loring, D.W., \& Fischer, J.S. (2012). Neuropsychological assessment. New York, NY: Oxford University Press.

Markela-Lerenc, J., Ille, N., Kaiser, S., Fiedler, P., Mundt, C., \& Weisbrod, M. (2004). Prefrontal-cingulate activation during executive control: which comes first? Cognitive Brain Research, 18(3), 278-287.

Mesulam, M.M. (2000). Principles of behavioral and cognitive neurology. Oxford: Oxford University Press.

Nicholson, G. \& Corbett, A. (1996). Slowing of central conduction in X-linked Charcot-Marie-Tooth neuropathy shown by brain stem auditory evoked responses. Journal of Neurology Neurosurgery and Psychiatry, 61(1), 43-46.

Nieuwenhuys, R., Voogd, J., \& van Huijzen, C. (2008). The human nervous system: a synopsis and atlas. Berlin: Srpinger-Verlag.

Panas, M., Kalfakis, N., Karadimas, C., \& Vassilopoulos, D. (2001). Episodes of generalized weakness in two sibs with the C164T mutation of the connexin 32 gene. Neurology, 57(10), 1906-1908. 
Pareyson, D. \& Marchesi, C.(2009). Diagnosis, natural history, and management of Charcot-Marie-Tooth disease. Lancet Neurology, 8(7), 654-667.

Paulson, H.L., Garbern, J.Y., Hoban, T.F., Krajewski, K.M., Lewis, R.A., Fischbeck, K.H., Grossman, R.I., Lenkinski, R., Kamholz, J.A., \& Shy, M.E. (2002), Transient central nervous system white matter abnormality in X-linked Charcot-Marie-Tooth disease. Annals of Neurology, 52(4), 429-434.

Petrides, M. (2014). Neuroanatomy of language regions of the human brain, 1st ed. New York: Academic Press.

Ribas, E.C., Yağmurlu, K., de Oliveira, E., Ribas, G.C., \& Rhoton, A. (2018). Microsurgical anatomy of the central core of the brain. Journal of Neurosurgery, 129(3), 752-769.

Rossor, A.M., Polke, J.M., Houlden, H., \& Reilly, M.M. (2013). Clinical implications of genetic advances in Charcot-MarieTooth disease. Nature Reviews Neurology, 9(10), 562-571.

Rottschy, C., Langner, R., Dogan, I., Reetz, K., Laird, A.R., Schulz, J.B., Fox, P.T., \& Eickhoff, S.B. (2012). Modelling neural correlates of working memory: a coordinate-based meta-analysis. Neuroimage, 60(1), 830-846.

Scherer, S.S. \& Kleopa, K.A. (2012). X-linked Charcot-Marie-Tooth disease. Journal of the Peripheral Nervous System, 17(s3), 9-13.

Shaywitz, B.A., Shaywitz, S.E., Pugh, K.R., Mencl, W.E., Fulbright, R.K., Skudlarski, P., Constable, R.T., Marchione, K.E., Fletcher, J.M., Lyon, G.R., \& Gore, J.C. (2002). Disruption of posterior brain systems for reading in children with developmental dyslexia. Biological psychiatry, 52(2), 101-110.

Shy, M.E., Blake, J., Krajewski, K.E.A., Fuerst, D.R., Laura, M., Hahn, A.F., Li, J., Lewis, R.A., \& Reilly, M. (2005). Reliability and validity of the CMT neuropathy score as a measure of disability. Neurology, 64(7), 1209-1214.

Simos, P.G., Breier, J.I., Fletcher, J.M., Foorman, B.R., Castillo, E.M., \& Papanicolaou, A.C. (2002). Brain mechanisms for reading words and pseudowords: an integrated approach. Cerebral Cortex, 12(3), 297-305.

Simos, P.G., Kasselimis, D., Potagas, C., \& Evdokimidis, I. (2014). Verbal comprehension ability in aphasia: demographic and lexical knowledge effects. Behavioural Neurology, 2014, 258303.

Simos, P.G., Papastefanakis, E., Panou, T., \& Kasselimis, D. (2011). The Greek memory scale. Rethymno: Laboratory of Applied Psychology, University of Crete.

Simos, P.G., Sideridis, G.D., Kasselimis, D., \& Mouzaki, A. (2013). Reading fluency estimates of current intellectual function: demographic factors and effects of type of stimuli. Journal of the International Neuropsychological Society, 19(3), 355-361.
Simos, P.G., Kasselimis, D., \& Mouzaki, A. (2011a). Age, gender, and education effects on vocabulary measures in Greek. Aphasiology, 25(4), 475-491.

Simos, P.G., Kasselimis, D., \& Mouzaki, A. (2011b). Effects of demographic variables and health status on brief vocabulary measures in Greek. Aphasiology, 25(4), 492-504.

Stancanelli, C., Taioli, F., Testi, S., Fabrizi, G.M., Arena, M.G., Granata, F., Russo, M., Gentile, L., Vita, G., \& Mazzeo, A. (2012). Unusual features of central nervous system involvement in CMTX associated with a novel mutation of GJB1 gene. Journal of the Peripheral Nervous System, 17(4), 407-411. https://doi. org/10.1111/j.1529-8027.2012.00439.x

van Paassen, B., van der Kooi, A., van Spaendonck-Zwarts, K., Verhamme, C., Baas, F., \& de Visser, M. (2014). PMP22 related neuropathies: Charcot-Marie-Tooth disease type $1 \mathrm{~A}$ and Hereditary Neuropathy with liability to Pressure Palsies. Orphanet Journal of Rare Diseases, 9(1), 38.

Vogt, B., Finch, D., \& Olson, C. (1992). Functional heterogeneity in cingulate cortex: the anterior executive and posterior evaluative regions. Cerebral Cortex, 2(6), 435-443.

Wager, T.D., \& Smith, E.E. (2003). Neuroimaging studies of working memory. Cognitive, Affective, \& Behavioral Neuroscience, 3(4), 255-274.

Wang, Y. \& Yin, F. (2016). A review of X-linked Charcot-MarieTooth disease. Journal of Child Neurology, 31(6), 761-772.

Zahr, N.M., Rohlfing, T., Pfefferbaum, A., \& Sullivan, E.V. (2009). Problem solving, working memory, and motor correlates of association and commissural fiber bundles in normal aging: A quantitative fiber tracking study. Neuroimage, 44(3), 10501062.

Zakzanis, K.K., Mraz, R., \& Graham, S.J. (2005). An fMRI study of the trail making test. Neuropsychologia, 43(13), 1878-1886.

Zalonis, I., Christidi, F., Bonakis, A., Kararizou, E., Triantafyllou, N.I., Paraskevas, G., Kapaki, E., \& Vasilopoulos, D. (2009). The stroop effect in Greek healthy population: Normative data for the Stroop Neuropsychological Screening Test. Archives of Clinical Neuropsychology, 24(1), 81-88.

Zalonis, I., Kararizou, E., Triantafyllou, N.I., Kapaki, E., Papageorgiou, S., Sgouropoulos, P., \& Vassilopoulos, D. (2007). A normative study of the trail making test A and B in greek adults. Clinical Neuropsychologist, 22(5), 842-850.

Zambelis, T., Panas, M., Kokotis, P., Karadima, G., Kararizou, E., \& Karandreas, N. (2008). Central motor and sensory pathway involvement in a X-linked Charcot-Marie-Tooth family. Acta Neurologica Belgica, 108(2), 44. 